C. Raab, M. Simkó*, U. Fiedeler, M. Nentwich, A. Gazsó

\section{Herstellungsverfahren von Nanopartikeln und Nanomaterialien}

\section{Zusammenfassung}

Materialien im Nanogrößenbereich werden schon seit vielen Jahrzehnten hergestellt, z. B. wird Carbon black (Industrieruß) in der Reifenindustrie verwendet. Dennoch vergrößert sich das Herstellungspotenzial durch die neuen und verbesserten Technologien von Jahr zu Jahr. Es handelt sich bei den meisten synthetisch hergestellten Nanomaterialien um Nanopartikel (80\%). Je nach Einsatzgebiet der Nanopartikel ist eine genau definierte Größenverteilung erforderlich, die jedoch von der chemischen Natur der Nanopartikel abhängen kann. Daher gibt es unterschiedliche Herstellungsverfahren von Nanopartikeln, um eine entsprechende GröBenverteilung zu erreichen. Verfahren in Lösungen oder Verfahren der Selbstorganisierung dürften am häufigsten zur Anwendung kommen. In diesem Dossier werden einige Verfahren zu Herstellung von Nanopartikeln vorgestellt.

\section{Einleitung}

Das Spektrum der Anwendungsbereiche von Nanomaterialien und/oder Nanopartikeln ist breit. Mittlerweile sind sie in vielen Produkten und in den verschiedensten Anwendungsbereichen vorhanden. Das Gros der großtechnisch gefertigten Nanoprodukte sind Nanopartikel, sie entstehen aber auch als Nebenprodukte bei der Herstellung anderer Materialien?.

Je nach Einsatzgebiet der Nanopartikel ist eine genau definierte und enge Partikelgrößenverteilung (Monodispersität) erforderlich. Mit Hilfe bestimmter Syntheseverfahren können gezielt Nanomaterialien hergestellt werden, wie Partikel, Partikel in dünnen Schichten, Dispersionen oder Verbundmaterialien etc.. Für das Erreichen der größenabhängigen Partikeleigenschaften sind Herstellungs- und Reaktionsbedingungen von ausschlaggebender Bedeutung. Größe, chemische Zusammensetzung, Kristallinität und Form der Partikel lassen sich über Temperatur, pH-Wert, Konzentration, chemische Zusammensetzung, Oberflächenmodifikation und Prozessführung steuern.
Für die Herstellung von Nanopartikel werden grundsätzlich zwei verschiedene Strategien verfolgt: „Top-Down" und "BottomUp". Unter "Top-Down" (von oben nach unten) versteht man im Zusammenhang mit Nanoparikeln die mechanische Zerkleinerung des Ursprungsmaterials mittels eines Mahlprozesses. In der "Bottom-Up" Strategie (von unten nach oben) werden Strukturen mittels chemischer Prozesse aufgebaut (Abbildung 1). Die Auswahl des jeweiligen Verfahrens richtet sich nach der chemischen Zusammensetzung und den anvisierten Eigenschaften der herzustellenden Nanopartikel.

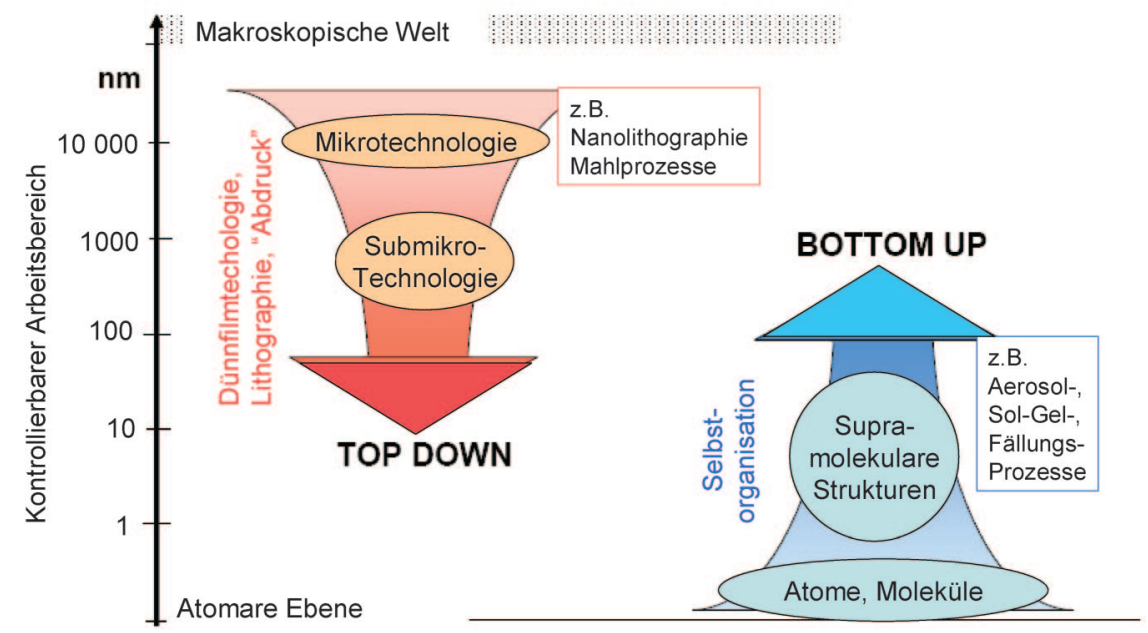




\section{Top-Down/Mechanisch- physikalische Herstellungsverfahren}

Unter "Top-Down" werden mechanisch-physikalische Herstellungsverfahren verstanden, die auf Ansätzen der Mikrosystemtechnik beruhen. Als gängigste mechanischphysikalische Zerkleinerungsmethode für die Herstellung von Nanopartikeln kommen verschiedene Mahlprozesse in Frage, die dem Top-Down Verfahren zugerechnet werden (Abbildung 2).

\section{Mahlprozesse}

Im mechanischen Herstellungsansatz werden Mikropartikel durch Mahlen zerkleinert. Mahlverfahren kommen bei der Herstellung metallischer und keramischer Nanomaterialien zum Einsatz. Für die Herstellung metallischer Nanopartikel werden herkömmliche Ausgangsstoffe (wie etwa Metalloxide) mithilfe von Hochenergie-Kugelmühlen pulverisiert. Die Hochenergie-Kugelmühlen sind hierzu mit Mahlkörpern aus Wolframcarbid oder Stahl ausgerüstet.

Mahlen ist mit thermischer Belastung und Energieaufwand verbunden. Ein etwaiger Abrieb der Mahlkörper bei langen Einsatzzeiten kann zur Verunreinigung der Partikel führen. Neben der rein mechanischen Vermahlung kann auch die Reaktivmahlung zum Einsatz kommen, bei der während des Mahlprozesses gleichzeitig eine chemische bzw. chemisch-physikalische Reaktion abläuft.

Verglichen mit den chemisch-physikalischen Herstellungsverfahren (s. u.) führt die Zerkleinerung von Partikeln durch Mahlen zu Produktpulvern mit relativ breiter PartikelgröBenverteilung. Eine gezielte Kontrolle der Partikelform ist mit dieser Methode praktisch nicht möglich.

\section{Bottom-Up/ Chemisch-physikalische Herstellungsverfahren}

Bottom-Up-Methoden nutzen die physikalischchemischen Grundsätze der molekularen bzw. atomaren Selbstorganisation. In diesen Herstellungsverfahren werden komplexere Strukturen gezielt aus Atomen bzw. Molekülen aufgebaut, wodurch die Kontrolle der Partikelgröße und Form sowie der Partikelgrößenverteilung ermöglicht wird. Darunter fallen zum Beispiel Aerosolverfahren, Fällungsreaktionen und Sol-Gel-Prozesse (Abbildung 3).

\section{Gasphasenprozesse (Aerosolprozesse) ${ }^{1}$}

Gasphasenprozesse gehören zu den großtechnisch gängigsten Verfahren, um Nanomaterialien in Pulver- oder in Filmform herzustellen.

Nanopartikel werden aus der Gasphase gebildet, indem ein Dampf des Produktmaterials auf chemischem oder physikalischem Wege erzeugt wird. Die Bildung erster Nanopartikel, die sich im flüssigen oder festen Zustand befinden können, erfolgt durch homogene Keimbildung. Das weitere Partikelwachstum findet je nach Prozess durch Kondensation (Übergang vom gasförmigen in den flüssigen Aggregatzustand), durch chemische Reaktion(en) an der Partikeloberfläche und/oder durch Koagulationsvorgänge (Aneinanderhaftung zweier oder mehrere Partikel) sowie durch Koaleszenzvorgänge (Fusion von Partikeln) statt. Beispiele sind Verfahren in Flammen-, Plasma-, Laser- und

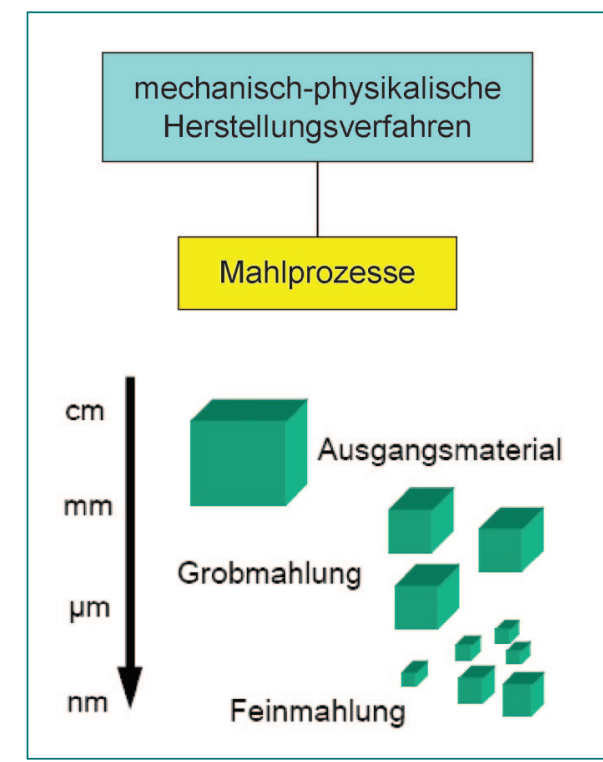

Abbildung 2:

Übersicht über mechanisch-physikalische Verfahren der Nanopartikelherstellung

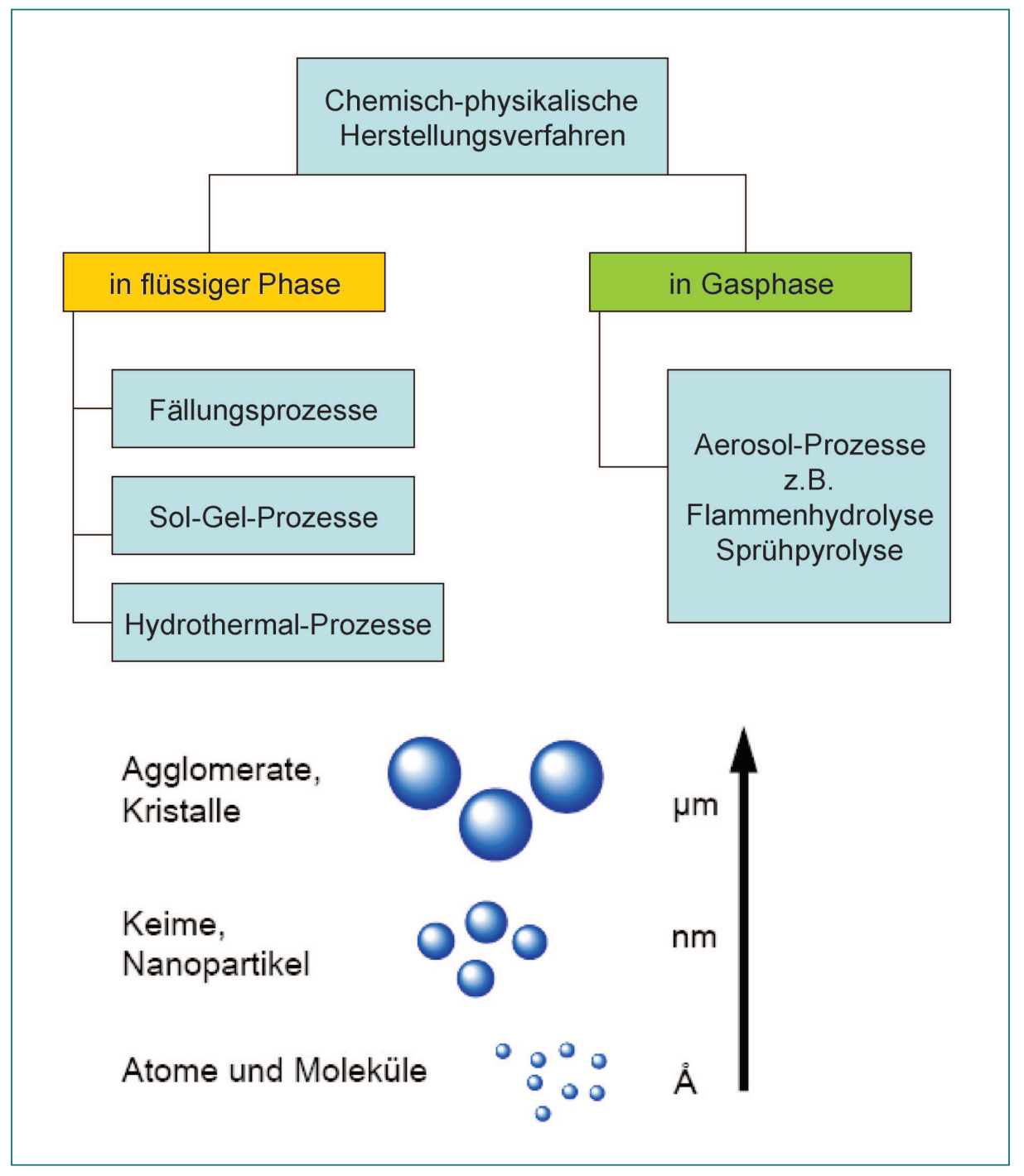

Abbildung 3: Chemisch-physikalische Prozesse zur Nanopartikelherstellung 
Heißwandreaktoren, wie sie für die die Herstellung von Fullerene und Kohlenstoffnanoröhrchen verwendet werden:

- In Flammenreaktoren werden Nanopartikel durch die Zersetzung von Ausgangsmolekülen in der Flamme bei relativ hohen Temperaturen (etwa $1.200-2.200^{\circ} \mathrm{C}$ ) gebildet. Flammenreaktoren werden heute großtechnisch für die Herstellung von Ruß, Pigment-Titandioxid und Siliziumdioxidpartikel verwendet.

- In Plasmareaktoren liefert Plasma (ionisiertes Gas) die Energie für die Verdampfung und zur Initialisierung von Zersetzungsreaktionen.

- In Laserreaktoren wird der gasförmige Ausgangsstoff mit einem Laser selektiv über die Absorptionswellenlänge erhitz† und zum gewünschten Produkt zersetzt.

- In Heißwandreaktoren wird Verdampfung und Kondensation eingesetzt. Das Ausgangsmaterial wird bei niedrigem Drücken (um $1 \mathrm{mBar}$ ) in ein reaktionsträges Gas verdampft, das die angereicherte Gasphase aus der heißen Zone entfernt. Die durch rasches Abkühlen gebildeten Partikel werden auf Filtern gesammelt. Technisch werden Heißwandreaktoren beispielsweise für die Herstellung von nanoskalierten Nickel- und Eisenpulvern verwendet.

- Das chemische Gasphasenabscheidungsverfahren wird für die direkte Abscheidung der Nanopartikel aus der Gasphase auf die Oberflächen verwendet. Dabei wird der Ausgangsstoff im Vakuum verdampft und an einer erhitzten Oberfläche in einer chemischen Reaktion kondensiert, d. h. aus der Gasphase in das feste Endprodukt abgeschieden.

\section{Tropfenbildung aus Partikel}

Partikel können mit Hilfe von Fliehkraft, Druckluft, Schall, Ultraschall, Vibrationen, Elektrostatik und anderen Methoden auch aus Tropfen gebildet werden. Die Tropfen werden entweder durch direkte Pyrolyse (thermische Spaltung chemischer Verbindungen) oder durch direkte Reaktionen mit einem anderen Gas in Pulver umgewandelt. Bei der Sprüh-Pyrolyse werden Tropfen des Ausgangsstoffes durch ein Hochtemperaturfeld (Flamme, Ofen) transportiert, was zur raschen Verdampfung der leicht flüchtigen Komponente oder zu Zersetzungsreaktionen führt. Die gebildeten Partikel werden auf Filtern gesammelt.

\section{Flüssigphasenprozesse ${ }^{3}$}

Die nasschemische Synthese von Nanomaterialien läuft in der Regel bei niedrigeren Temperaturen als die Gasphasensynthese ab. Die wichtigsten Flüssigphasenprozesse zur Herstellung von Nanomaterialien sind Fällungen, Sol-Gel-Prozesse und Hydrothermal-Synthesen (siehe Abbildung 3).

\section{Fällungsprozesse}

Die Fällung (Ausflockung) von Feststoffen aus einer Metallionen enthaltenden Lösung ist eines der am häufigsten verwendeten Herstellungsverfahren von Nanomaterialien. Metalloxide, nichtoxidische und auch metallische Nanopartikel können so durch Fällung hergestellt werden. Das Verfahren basiert auf Reaktionen von in Lösungsmitteln gelösten Ausgangssalzen. Zur Bildung des gewünschten Partikelniederschlags wird ein Fällungsreagenz zugegeben und der gebildete Niederschlag abfiltriert und thermisch nachbehandelt.

Bei Fällungsprozessen werden Partikelgröße und -größenverteilung, Kristallinität und Morphologie (Form) durch die Reaktionskinetik (Reaktionsgeschwindigkeit) bestimmt. Neben der Konzentration der Ausgangsstof$\mathrm{fe}$, zählen die Temperatur, der pH-Wert der Lösung und auch die Reihenfolge der Zugabe der Ausgangsstoffe und Mischvorgänge zu den Einflussfaktoren.

Für eine gute Größenkontrolle kann die Verwendung von selbstanordnenden Membranen erreicht werden, die als Nanoreaktoren zur Partikelherstellung dienen. Zu diesen Nanoreaktoren gehören Mikroemulsionen, Blasen, Mizellen und Liposome. Sie bestehen aus einer polaren Gruppe und einer unpolaren Kohlenwasserstoffkette. Mikroemulsionen bestehen zum Beispiel aus zwei, in den verwendeten Konzentrationen nicht miteinander mischbaren Flüssigkeiten, meistens Wasser und Öl und mindestens einem Tensid (Substanz welche die Oberflächenspannung von Flüssigkeiten herabsetzt). In bestimmten Lösungsmitteln formen sich so kleine Reaktionsräume, in denen Keimbildung und kontrolliertes Partikelwachstum vonstatten gehen. Die Partikelgröße wird durch die Größe der Nanoreaktoren bestimmt und gleichzeitig wird die Agglomeration (Verklumpung) der Partikel verhindert. Mikroemulsionsverfahren kommen insbesondere bei der Herstellung von Nanopartikeln mit bestimmten Funktionen für Pharmazeutika und Kosmetika zum Einsatz.
Ein weiteres Verfahren, das auf selbstorganisiertem Wachstum mit Templaten (schablonartige Abstandhalter) basiert, ist die Hydrothermal-Synthese. Zeolithe (mikroporöse Aluminium-Silizium-Verbindungen) werden aus wässrig überhitzen Lösungen in Autoklaven (luftdicht verschließbaren Druckbehältern) hergestellt. Durch das teilweise Verdampfen des Lösungsmittels baut sich im Autoklaven ein Druck (von einigen Bar) auf, wodurch andere chemische Reaktionen ablaufen als unter Standardbedingungen, z. B. durch die Veränderung der Löslichkeiten. Die Bildung von Nanopartikeln und die Form der Hohlräume kann durch Zugabe von Templaten gesteuert werden. Template sind Partikel mit Verbindungen, die die Bildung von bestimmten Formen und Größen ermöglichen.

\section{Sol-Gel-Prozesse}

Sol-Gel-Synthesen (Herstellung eines Gels aus pulverförmigen Stoffen) sind nasschemische Verfahren zur Herstellung poröser Nanomaterialien, keramisch nanostrukturierter Polymere und Beschichtungen sowie oxidischer Nanopartikel. Die Synthese läuft unter relativ milden Bedingungen und niedrigen Temperaturen ab. Als Sole werden Dispersionen fester Partikel im Größenbereich zwischen 1-100 nm bezeichnet, die sich fein verteilt in Wasser oder organischen Lösungsmitteln befinden.

Beim Sol-Gel-Verfahren erfolgt die Herstellung bzw. Abscheidung der Werkstoffe jeweils von einem flüssigen Sol-Zustand aus, der durch eine Sol-Gel-Transformation in einen festen Gel-Zustand überführt wird. Während der Sol-Gel-Umwandlung kommt es zu einer drei-dimensionalen Vernetzung der Nanopartikel im Lösungsmittel, wodurch das Gel Festkörpereigenschaften erhält. Gele lassen sich z. B. in einem (oxid)keramischen Werkstoff durch eine kontrollierte Wärmebehandlung unter Luft umformen.

Zunächst kann eine Lösung aus einem Alkoxid (Metallverbindung eines Alkohols z. B. mit Silizium, Titan oder Aluminium) eine organometallische Verbindung durch die Zugabe von organischen Stoffen im Sol-Gel Prozess hergestellt werden. Der pH-Wert der Lösung wird mit einer Säure oder Base eingestellt, die auch als Katalysator die Umsetzung des Alkoxids auslöst. Die ablaufenden Reaktionen sind Hydrolyse (Spaltung einer chemischen Verbindung durch Wasser) und anschließende Kondensation und Polymerisation (Reaktion aus eingliedrigen zu vieloder langgliedrigen Verbindungen). Die Partikel oder das oxidische Polymer wachsen 
bei fortlaufender Reaktion, bis ein Gel entsteht. Bedingt durch die hohe Porosität des Netzwerkes haben die Partikel meist eine große Oberfläche, einige hundert Quadratmeter pro Gramm. Der Ablauf der Hydrolyse und Polykondensationsreaktion hängt von vielen Faktoren ab: Zusammensetzung der Ausgangslösung, Art und Menge des Katalysators, Temperatur sowie Reaktor- und Mischgeometrie.

Die alkoxidische Ausgangslösung des SolGel-Prozesses kann für Beschichtungen auch auf Oberflächen beliebiger Geometrie aufgebracht werden. Nach der Benetzung erfolgt durch die Gel-Bildung der Aufbau des porösen Netzwerkes, was zu Schichtdicken von 50-500 nm führt. Dickere Schichten, die zum Beispiel als Membranen eingesetz† werden können, entstehen durch wiederholtes Benetzen und Trocknen. Auch die Herstellung von Fasern ist durch das Sol-GelVerfahren möglich. An die Bildung des Gels schließt sich in allen Fällen ein Trocknungsschritt an. Abbildung 4 verdeutlicht die unterschiedlichen Reaktions- und Verarbeitungsschritte des Sol-Gel-Prozesses.

Ein bedeutender Vorteil des Sol-Gel-Verfahrens liegt in der Verarbeitbarkeit der Sole und Gele, je nach Behandlungsschritt zu Pulvern, Fasern, Keramiken und Beschichtungen. Außerdem können hochporöse Nanomaterialien hergestellt werden. Durch Füllung dieser Poren während oder nach der Gelherstellung lassen sich Verbundwerkstoffe herstellen. Die tiefe Prozesstemperatur gestattet es ferner, Wirkstoffe im Laufe des Syntheseschrittes in das Gel einzubetten, die gespeichert und kontrolliert wieder freigesetz† werden können. Die Nachteile des Sol-GelProzesses liegen bei den schwer zu kontrollierenden Synthese- und Trocknungsschritten, die ein Scale-up des Verfahrens erschweren. Ferner können organische Verunreinigungen im Gel verbleiben. Die meist notwendigen Reinigungsschritte, Trocknung und thermische Nachbehandlung führen zu einem im Vergleich zur Gasphasensynthese aufwändigen Herstellungsprozess.

Die nasschemische Synthese von Nanomaterialien führt dazu, dass die gewünschten kristallinen Formen oftmals nicht eingestellt werden können und die thermische Stabilität der Produktpulver geringer ist. Eine thermische Nachbehandlung mit wiederholter Verminderung der Oberfläche der Partikel ist in diesem Fall notwendig. Andererseits lassen sich über die Flüssigphase hochporöse Materialien produzieren, die aufgrund der hohen Temperaturen in Gasphasenreaktoren meist nicht herstellbar sind. Mit einigen Ausnahmen lassen Gasphasenpro- zesse auch nicht die Produktion organischer Nanopartikel zu. Flüssigphasenprozesse eignen sich besonders für die gezielte Herstellung monodisperser Produktpulver (mit einheitliche Partikelgröße).

\section{Erläuterungen:}

Dispersion (disperses System): Aus zwei oder mehreren Phasen bestehendes Stoffgemisch, bei dem ein Stoff in einem anderen in feinster Form verteilt ist.

Emulsion: Disperses System, bei dem Flüssigkeitströpfchen in einer anderen Flüssigkeit verteilt sind.

Suspension: Disperses System, bei dem unlösliche Feststoffteilchen in einer Flüssigkeit verteilt sind.

\section{Anmerkungen und Literaturhinweise}

1 Rössler A, Georgios Skillas Sotiris E. Pratsinis, 2001, Nanopartikel - Materialien der Zukunft: Maßgeschneiderte Werkstoffe, Chemie in unserer Zeit 35(1), 32-41.

2 Imn.web.psi.ch.

3 Stefan, E., 2004, Chemische Technik. Prozesse und Produkte, 5. Aufl. Herausgegeben von Roland Dittmeyer, Wilhelm Keim, Gerhard Kreysa und Alfred Oberholz, Angewandte Chemie 116(42), 5.687-5.788.

4 www.uni-ulm.de/uni/fak/natwis/anorgchem.

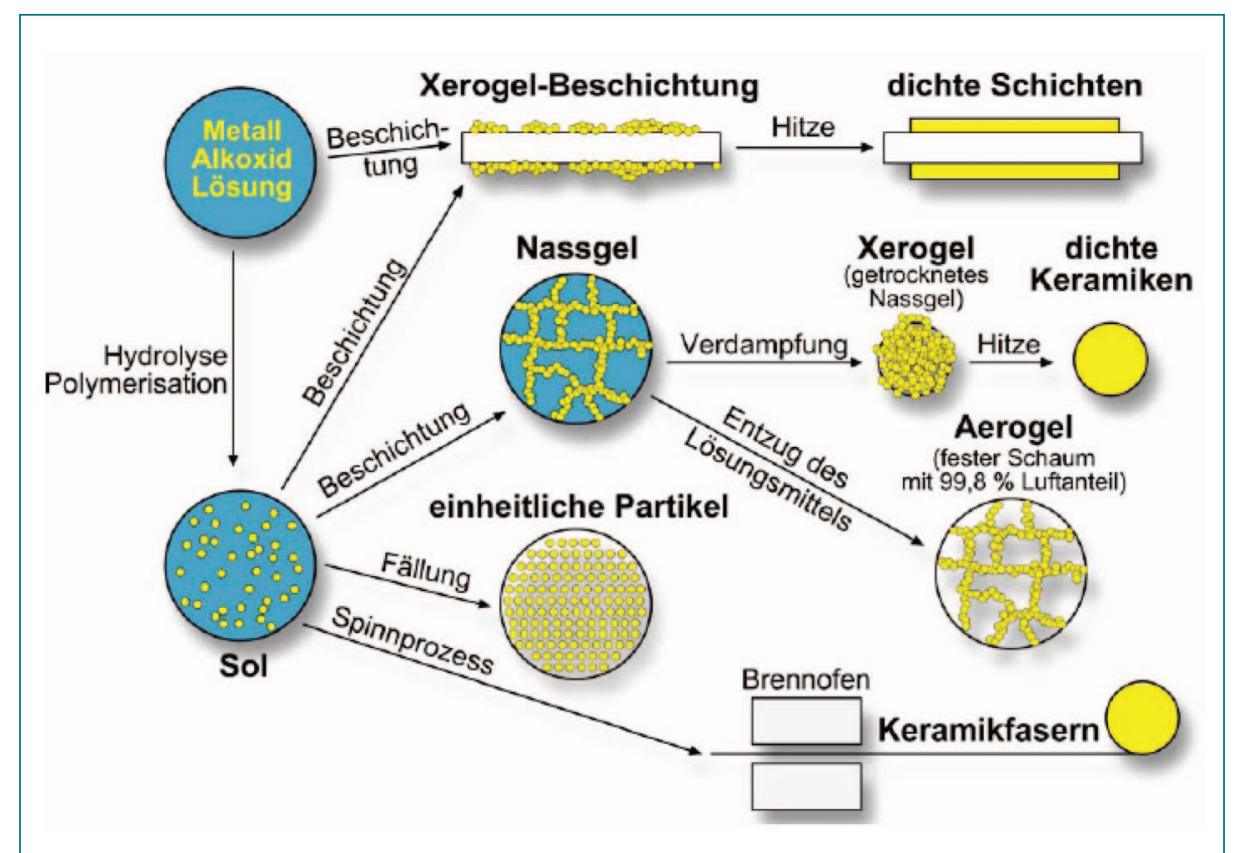

\section{IMPRESSUM:}

Medieninhaber: Österreichische Akademie der Wissenschaften; Juristische Person öffentlichen Rechts (BGBI 569/1921 idF BGBI I 130/2003); Dr. Ignaz Seipel-Platz 2, A-1010 Wien

Herausgeber: Institut für Technikfolgen-Abschätzung (ITA); Strohgasse 45/5, A-1030 Wien; www.oeaw.ac.at/ite

Erscheinungsweise: Die NanoTrust-Dossiers erscheinen unregelmäßig und dienen der Veröffentlichung der Forschungsergebnisse des Instituts für Technikfolgen-Abschätzung im Rahmen des Projekłs NanoTrust. Die Berichte werden ausschließlich über das Internetportal „epub.oeaw” der Öffentlichkeit zur Verfügung gestellt: epub.oeaw.ac.at/ita/nanotrust-dossiers/

NanoTrust-Dossier Nr. 006 November 2008: epub.oeaw.ac.at/ita/nanotrust-dossiers/dossier006.pdf ISSN: 1998-7293 